\title{
In Vivo High Frame Rate Vector Flow Imaging Using Plane Waves and Directional Beamforming
}

Jensen, Jonas; Villagómez Hoyos, Carlos Armando; Stuart, Matthias Bo; Ewertsen, Caroline; Nielsen, Michael Bachmann; Jensen, Jørgen Arendt

\section{Published in:}

Proceedings of IEEE International Ultrasonics Symposium,

Link to article, DOI:

10.1109/ULTSYM.2016.7728662

Publication date:

2016

Document Version

Peer reviewed version

Link back to DTU Orbit

Citation (APA):

Jensen, J., Villagómez Hoyos, C. A., Stuart, M. B., Ewertsen, C., Nielsen, M. B., \& Jensen, J. A. (2016). In Vivo High Frame Rate Vector Flow Imaging Using Plane Waves and Directional Beamforming. In Proceedings of IEEE International Ultrasonics Symposium, IEEE. https://doi.org/10.1109/ULTSYM.2016.7728662

\section{General rights}

Copyright and moral rights for the publications made accessible in the public portal are retained by the authors and/or other copyright owners and it is a condition of accessing publications that users recognise and abide by the legal requirements associated with these rights.

- Users may download and print one copy of any publication from the public portal for the purpose of private study or research.

- You may not further distribute the material or use it for any profit-making activity or commercial gain

- You may freely distribute the URL identifying the publication in the public portal 


\title{
In Vivo High Frame Rate Vector Flow Imaging Using Plane Waves and Directional Beamforming
}

\author{
Jonas Jensen ${ }^{1}$, Carlos Armando Villagómez Hoyos ${ }^{1}$, Matthias Bo Stuart ${ }^{1}$, Caroline Ewertsen ${ }^{2}$, \\ Michael Bachmann Nielsen ${ }^{2}$, and Jørgen Arendt Jensen ${ }^{1}$ \\ ${ }^{1}$ Center for Fast Ultrasound Imaging, Department of Electrical Engineering, \\ Technical University of Denmark, DK-2800 Lyngby, Denmark \\ ${ }^{2}$ Department of Radiology, Copenhagen University Hospital, DK-2100 Copenhagen, Denmark
}

\begin{abstract}
Directional beamforming (DB) estimates blood flow velocities accurately when the flow angle is known. However, for automatically finding the flow angle a computationally expensive approach is used. This work presents a method for estimating the flow angle using a combination of inexpensive transverse oscillation (TO) estimators and only 3 directional beamformed lines. The suggested DB vector flow estimator is employed with steered plane wave transmissions for high frame rate imaging. Two distinct plane wave sequences are used: a short sequence (3 angles) for fast flow and an interleaved long sequence (21 angles) for both slow flow and B-mode. Parabolic flow with a peak velocity of $0.5 \mathrm{~m} / \mathrm{s}$ is measured at beam-to-flow angles of $60^{\circ}$ and $90^{\circ}$. The DB method estimates the angle with a bias and standard deviation (STD) less than $2^{\circ}$, and the STD of the velocity magnitude is $2.5 \%$. This is $7-8.5 \%$ when using TO. The long sequence has a higher sensitivity, and when used for estimation of slow flow with a peak velocity of $0.04 \mathrm{~m} / \mathrm{s}$, the SD is $2.5 \%$ and bias is $0.1 \%$. This is a factor of 4 better than if the short sequence is used. The carotid bifurcation was scanned on a healthy volunteer, and the short sequence was used with TO and DB to estimate velocity vectors. The STD of the velocity profile over a cardiac cycle was $6.1 \%$ for TO and $4.9 \%$ for DB.
\end{abstract}

\section{INTRODUCTION}

Ultrasound blood flow estimation is of diagnostic value in investigating hemodynamics in the human cardiovascular system. Conventional color flow mapping uses focused emissions in line-by-line imaging, where each line is acquired sequentially from received echoes of several consecutive pulses. It limits the frame rate significantly, and full flow dynamics of both fast and slow flow are lost, because of the limited observation time along each focusing direction.

Plane wave imaging is a synthetic aperture technique, which can increase the frame rate and improve image quality [1]. An image of the entire insonified region is created for each emission resulting in a low-resolution image (LRI). By using a few emissions, the low-resolution images can be summed to form a high-resolution image (HRI).

Vector flow imaging methods reveal the 2-D velocity vector without the need for angle correcting conventional 1D estimates. Transverse Oscillation (TO) [2] and directional beamforming (DB) [3] are two methods capable of estimating $2-\mathrm{D}$ velocity vectors. The DB approach uses focusing along a line following the direction of the flow. The velocity magnitude is estimated accurately using a cross-correlation estimator, and it has also been shown that the standard deviation of the velocity estimates can be reduced by a factor 2 , when using DB rather than TO [4]. However, the direction of the blood flow needs to be known in advance for DB [5]. Automatic approaches for angle estimation have been proposed [6], [7]. The number of calculations for these angle estimators are, however, very high, because signals have to be beamformed in a polar grid and cross-correlated at every angle and for each estimation point. If lines are beamformed at every $5^{\circ}$, the number of directional lines is 37 . Therefore, it is of interest to investigate angle estimators, which reduce the number of calculations.

This paper presents a method for DB, where the flow angle is found automatically using a combination of inexpensive TO estimators and DB. Only 3 lines are beamformed for each estimation point in addition to 2 lines for TO, thus, at least a sevenfold reduction in beamforming load is achieved compared to the current angle estimators. The method is a two-step procedure, where the initial TO estimate is followed by a DB estimate. The estimates obtained by TO and DB can, thus, be compared. Plane waves are used for high-frame rate imaging, and it is shown that both fast and slow flow can be estimated accurately, when interleaving a short and a long emission sequence.

\section{Method for Plane Wave Flow Imaging}

The proposed method for vector flow imaging uses steered plane waves in transmit and a two-step procedure for velocity estimation: First, TO estimators are used to find an initial flow angle, and then 3 directional lines are beamformed around the TO angle to improve the angle estimate. The velocity magnitude is estimated along the flow direction using crosscorrelation estimators. An illustration of the principle is shown in Fig. 1, where 3 directional lines are beamformed around the initial TO angle estimate.

\section{A. Transverse Oscillation}

A transverse oscillation in the pulse-echo field can be created in the receive beamforming by using a two-peak apodization function, or in the Fourier domain by filtering the beamformed image in the lateral dimension. The latter approach is used here, where a Gaussian filter is applied on the Fourier transformed HRIs to only select Fourier components around a desired lateral oscillation frequency [8], [9]. 


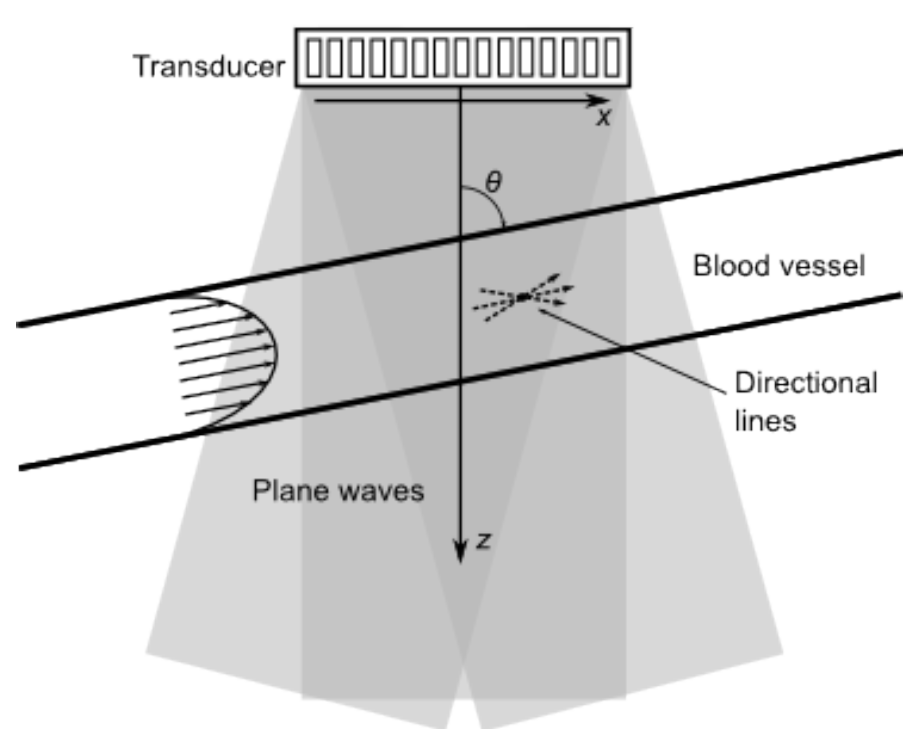

Fig. 1. Plane wave emissions from a linear array transducer are used to insonify flow in a straight vessel, which has a beam-to-flow angle $\theta$. Directional lines are beamformed around the initial TO angle estimate.

The directional information of the flow is preserved by applying the Hilbert transform on the TO image for each of the lines in the lateral direction. This avoids having a spatial quadrature between two beamformed signals [10]. The actual mean lateral oscillation frequency at a given depth is estimated directly from the data and used for the transverse velocity component $v_{x}$. For the axial velocity component $v_{z}$, a crosscorrelation estimator is employed.

The flow angle is found at each estimation point from:

$$
\theta=\arctan \left(v_{x}, v_{z}\right)
$$

using the estimated $v_{x}$ and $v_{z}$.

\section{B. Directional Beamforming}

The initial angle estimate from (1) is used to directionally beamform a signal $y_{d}(k)$ at an estimation point and at an angle $\theta$. Correlating signals from two HRI acquired $T_{p r f, \text { eff }}$ seconds apart gives

$$
R_{12}(l)=\frac{1}{N_{k}+1} \sum_{k=-N_{k} / 2}^{N_{k} / 2} y_{d}^{(t)}(k) y_{d}^{\left(t+T_{p r f, e f f}\right)}(k+l),
$$

where $y_{d}^{(t)}(k)$ is the directional signal focused at time $t$.

Beamforming signals at other angles $\theta_{m}=\theta \pm \Delta \theta$ yield correlation functions $R_{12}\left(l, \theta_{m}\right)$. They are used to calculate the normalized cross-correlation estimate

$$
R_{12 n}\left(\theta_{m}\right)=\left(\frac{\max \left(R_{12}\left(l, \theta_{m}\right)\right)}{R_{11}\left(0, \theta_{m}\right)}\right),
$$

which gives the maximum normalized cross-correlation for three angles. $R_{11}\left(0, \theta_{m}\right)$ is the power of the signal. The angle estimate, $\hat{\theta}$, is found as the angle yielding the largest correlation in (3). The estimate is also improved by interpolation.
TABLE I

PROCESSING PARAMETERS.

\begin{tabular}{lcc}
\hline Parameter & TO & DB \\
\hline Receive apodization & Tukey & Tukey \\
Receive F-number & 0.8 & 0.8 \\
Sampling interval $\Delta r$ & $\lambda / 20$ & $\lambda / 20$ \\
Desired lateral wavelength & $1.53 \mathrm{~mm}$ & - \\
TO window size & $1.5 \mathrm{~mm}$ & \\
Number of HRI/estimate & 32 & 32 \\
Line length & - & $12.5 \lambda$ \\
\hline
\end{tabular}

The angle estimate $\hat{\theta}$ is used to directional beamform a line for this angle. The velocity magnitude is found from the lag $l_{\text {max }}$, where $R_{12}(l, \hat{\theta})$ is maximum, and convert it to velocity

$$
|v|=\frac{l_{\max } \Delta r}{T_{p r f, e f f}} .
$$

\section{METHODS FOR EXPERIMENTS}

A short plane wave sequence $\left(-15^{\circ}, 0^{\circ}, 15^{\circ}\right)$ is used for fast flow estimation, and a long sequence (21 angles, max steering $20^{\circ}$ ) is used for both B-mode imaging and slow flow estimation. The sequences are interleaved in a $3+1$ procedure, i.e., one emission from the long sequence is transmitted after the short sequence. The pulse repetition frequency $f_{p r f}$ is $10 \mathrm{kHz}$, thus, the effective $f_{p r f, e f f}$ for the short sequence is $f_{p r f} / 4$ and $f_{p r f} / 84$ for the long sequence. A 1.5 -cycle excitation pulse is used in both sequences, since the precision of the cross-correlation estimator used for DB is proportional to the bandwidth of the system. To achieve narrow-band signals suitable for TO estimation, beamformed signals are convolved with a 4-cycle sinusoid.

Processing parameters are listed in Table I. Three directional lines are beamformed at each estimation point for DB: one at the TO angle $\theta$ and at $\pm \Delta \theta$. For constant flow, $\Delta \theta=1$ standard deviation (STD) of the TO angle estimate, which is calculated from 10 non-consecutive estimates at each estimation point. Due to pulsation of flow for the in vivo scan, lines are beamformed at a fixed $\Delta \theta=2.5^{\circ}$ around the $\mathrm{TO}$ angle in this case.

\section{A. Measurement Setup}

The approach is implemented on the experimental scanner SARUS [11] for acquisition of channel RF data. A 192element 4.1 MHz transducer is employed (pitch $0.6 \lambda$ ). A flow rig phantom with a tube radius of $6 \mathrm{~mm}$ and placed at a depth of $20 \mathrm{~mm}$ is scanned, and the beam-to-flow angle $\theta$ is $60^{\circ}$ and $90^{\circ}$. Constant parabolic flow is circulated and volume flow is measured by a magnetic flow meter for reference (MAG 3000, Danfoss, Nordborg, Denmark). The $f_{p r f}$ is reduced to $2 \mathrm{kHz}$ and the peak velocity is $0.1 \mathrm{~m} / \mathrm{s}$ to avoid turbulence (corresponds to fast velocity flow of $0.5 \mathrm{~m} / \mathrm{s}$ at $10 \mathrm{kHz} f_{p r f}$ ). Slow velocity flow is also measured on a flow phantom with a $3.5 \mathrm{~mm}$ tube radius and $90^{\circ}$ beam-to-flow angle. In this case the peak velocity is $0.04 \mathrm{~m} / \mathrm{s}$ and $f_{p r f}=10 \mathrm{kHz}$. Echocanceling is performed on beamformed data with a Hoeks filter [12]. 

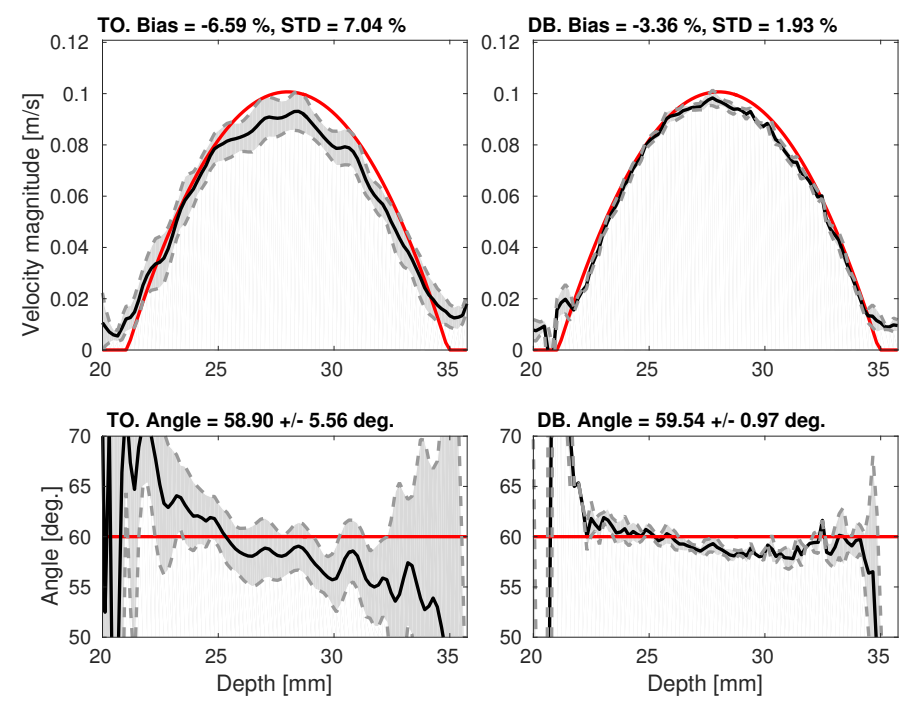

Fig. 2. Measurement on a straight vessel phantom at $60^{\circ}$ beam-to-flow-angle. Velocity magnitude (top) and angles (bottom) for TO (left) and DB (right). Mean estimates are shown in black with \pm 1 STD and true profiles in red.

The right carotid bifurcation on a 27-year old healthy male is scanned with a longitudinal view by an experienced radiologist. The scan sequence is the same as for simulation and phantom measurements, and $f_{p r f}$ is $10 \mathrm{kHz}$. Data are acquired for $4.5 \mathrm{~s}$. Echo-canceling is performed with a Hoeks filter for diastolic flow and an energy-based filter with manual threshold for systolic flow [13].

\section{RESUlts}

\section{A. Phantom Measurements}

Fig. 2 shows results from a flow rig measurement, where the beam-to-flow angle is $60^{\circ}$ and $f_{p r f}$ is $2 \mathrm{kHz}$. The short sequence is used for flow estimation. The mean velocity magnitude and angles are shown in black with \pm 1 STD, and the true velocities and angles are shown in red. Results from TO are shown in the left figures, while results for DB are shown in the right figures. The initial TO estimate gives a mean flow angle of $58.9 \pm 5.6^{\circ}$, which is improved in the DB step to $59.5 \pm 1^{\circ}$. The resulting velocity magnitude estimate is accurate with a mean bias of $-3.4 \%$, and the STD of the velocity reduces from $7 \%$ (TO) to $1.9 \%$ (DB). For the $90^{\circ}$ beam-to-flow angle measurement, the angle is estimated to $89.7^{\circ} \pm 1.3^{\circ}$ using TO and $89.8^{\circ} \pm 0.5^{\circ}$ using DB. The STD of the velocity magnitude is $8.5 \%$ for TO and $2.5 \%$ for DB, while bias changes slightly from $-3.9 \%$ for $\mathrm{TO}$ to $-5.4 \%$ for DB.

Results from the slow velocity flow measurement are shown in Fig. 3. The short sequence is used with the DB method for the result in the bottom figure, where the bias and STD are $-6 \%$ and $10 \%$, respectively. The performance can be improved by using the long sequence for flow estimation as shown in the top figure. The STD is reduced by a factor 4 . The long sequence has a higher sensitivity than the short sequence, because 21 rather than 3 LRI are combined, thus, reducing side-lobe levels and improving contrast. Estimates are correlated at every
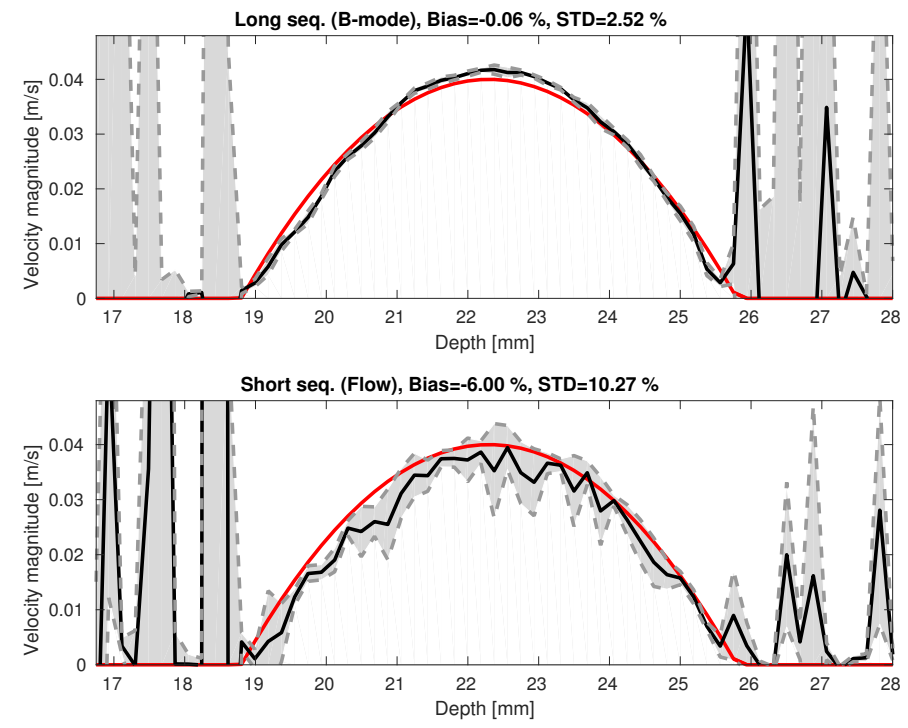

Fig. 3. Estimation of slow velocity flow using the long sequence (top) and short sequence (bottom). The beam-to-flow angle is $90^{\circ}$.

$8.4 \mathrm{~ms}$, which reduces $v_{x, \max }$ to $5.9 \mathrm{~cm} / \mathrm{s}$. Note that echocanceling has been performed for the same time period $(0.18$ s) for the two sequences. It is possible to use the slow flow sequence also for B-mode imaging, if a separate B-mode sequence cannot be included in the emission sequence. In that case, a compromise needs to be made for the receive gain.

\section{B. In Vivo Measurement}

A frame from the in vivo measurement of peak systole is shown in Fig. 4, which is processed using TO (left image) and DB (right image). The short sequence is used for flow estimation and the long for B-mode images. The direction of flow is indicated by arrows and follows the vessel anatomy. The DB method estimates larger velocities at the inlet of the external carotid artery, while low or no velocity is estimated in the top left corner of the flow image. This can be due to echo-canceling effects and/or angle estimation problems. The velocity magnitude at the indicated green point is shown as a function of time in Fig. 4 (bottom). After alignment of the profiles to the cardiac cycle, the STD for TO is calculated to $6.1 \%$ and $4.9 \%$ for DB.

\section{Discussion AND CONCLUSION}

A method for 2-D vector flow imaging, where the flow angle is found using a combination of TO and DB was presented. Compared to conventional DB methods, at least a sevenfold reduction in beamforming load is achieved for angle estimation. Rather than beamforming lines in a polar grid at all angles, the presented method uses an initial TO angle estimate to limit the angle search range and beamforms only 3 lines around the TO angle. It was demonstrated in phantom measurements that the method estimates flow angle accurately and with a bias and STD less than $2^{\circ}$. The STD of the velocity magnitude estimates was less than $2.5 \%$, which was an order of 2-3 times less than for the TO method. The 

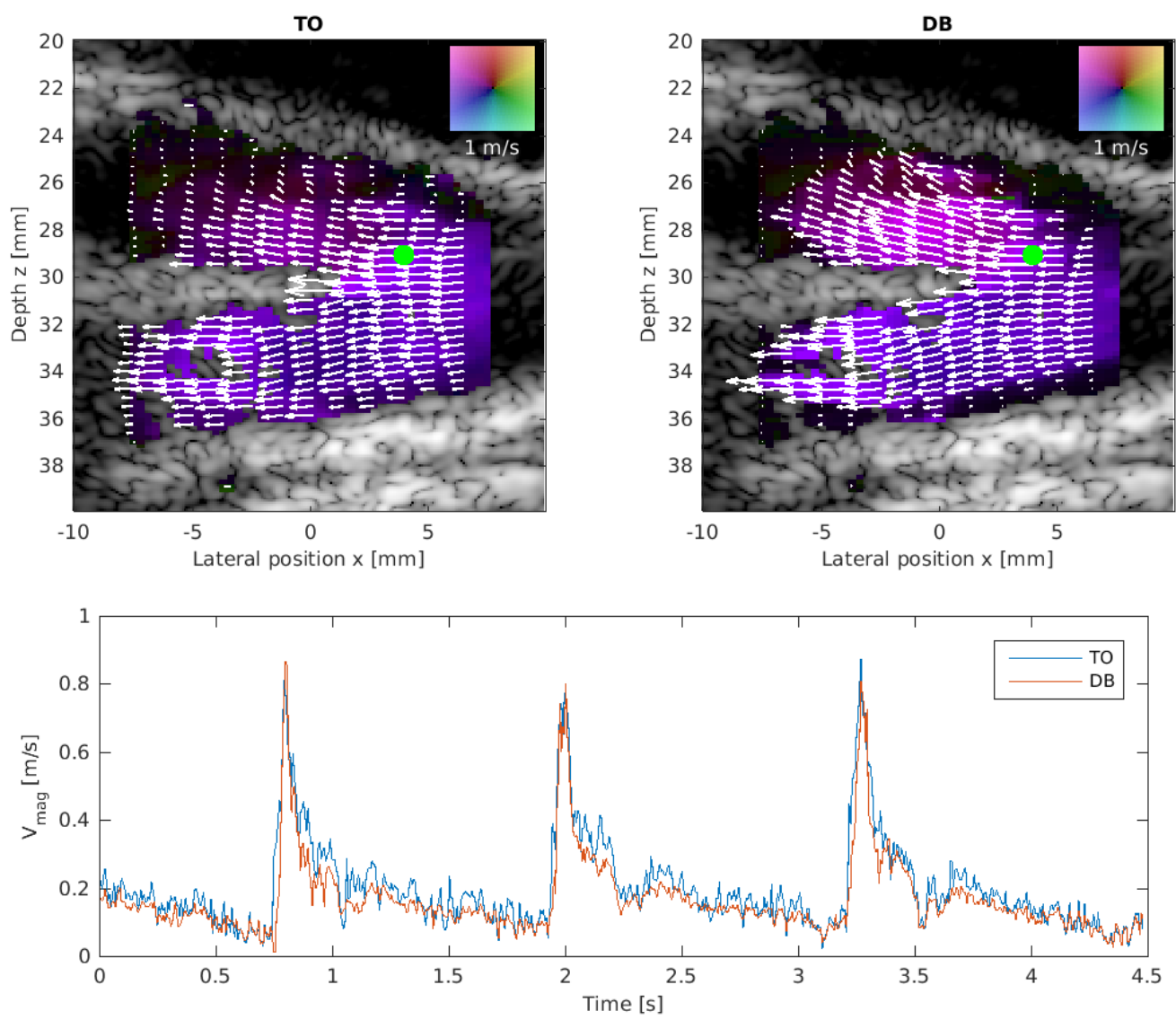

Fig. 4. Vector flow imaging of the carotid bifurcation. Flow in the systole is shown using TO in the left imaging and DB in the right image. The bottom figure shows velocity magnitude at a single estimation point (green point on VFI) as a function of time for the two methods.

method was employed with plane waves in transmit, and this acquisition scheme achieves a very high frame rate of 2000 fps for fast flow estimation. An interleaved long sequence was used for B-mode imaging and also used to improve slow flow estimation in a phantom. Potentially, this may improve slow and fast flow estimation, as it will be possible to use data simultaneously from the two sequences for flow estimation. It can also be an advantage for visualization of flow in both large and small vessels.

\section{ACKNOWLEDGMENTS}

This work was supported by grant 82-2012-4 from the Danish National Advanced Technology Foundation and by BK Ultrasound Aps.

\section{REFERENCES}

[1] M. Tanter, J. Bercoff, L. Sandrin, and M. Fink, "Ultrafast compound imaging for 2-D motion vector estimation: application to transient elastography," IEEE Trans. Ultrason., Ferroelec., Freq. Contr., vol. 49, pp. 1363-1374, 2002.

[2] J. A. Jensen and P. Munk, "A new method for estimation of velocity vectors," IEEE Trans. Ultrason., Ferroelec., Freq. Contr., vol. 45, pp. 837-851, 1998.

[3] J. A. Jensen, "Directional velocity estimation using focusing along the flow direction: I: Theory and simulation," IEEE Trans. Ultrason., Ferroelec., Freq. Contr., vol. 50, pp. 857-872, 2003.
[4] - "Comparison of vector velocity imaging using directional beamforming and transverse oscillation for a convex array transducer," in Proc. SPIE Med. Imag., vol. 9040, 2014, pp. 904 012-1-8.

[5] J. Udesen, F. Gran, and J. A. Jensen, "Fast Color Flow Mode Imaging Using Plane Wave Excitation and Temporal Encoding," in Proc. SPIE Med. Imag., vol. 5750, Feb. 2005, pp. 427-436.

[6] J. A. Jensen and N. Oddershede, "Estimation of velocity vectors in synthetic aperture ultrasound imaging," IEEE Trans. Med. Imag., vol. 25, pp. 1637-1644, 2006.

[7] C. A. Villagomez-Hoyos, M. B. Stuart, K. L. Hansen, M. B. Nielsen, and J. A. Jensen, "Accurate angle estimator for high frame rate 2-D vector flow imaging," IEEE Trans. Ultrason., Ferroelec., Freq. Contr., p. Accepted, 2016.

[8] S. Salles, A. J. Y. Chee, D. Garcia, A. C. H. Yu, D. Vray, and H. Liebgott, "2-D arterial wall motion imaging using ultrafast ultrasound and transverse oscillations," IEEE Trans. Ultrason., Ferroelec., Freq. Contr., vol. 62, no. 6, pp. 1047-1058, 2015.

[9] J. Jensen, M. B. Stuart, and J. A. Jensen, "High frame rate vector velocity estimation using plane waves and transverse oscillation," in Proc. IEEE Ultrason. Symp., 2015, pp. 1-4.

[10] J. A. Jensen, "Improved vector velocity estimation using directional transverse oscillation," in Proc. IEEE Ultrason. Symp. IEEE, 2015, pp. $1-4$.

[11] J. A. Jensen, H. Holten-Lund, R. T. Nilsson, M. Hansen, U. D. Larsen, R. P. Domsten, B. G. Tomov, M. B. Stuart, S. I. Nikolov, M. J. Pihl, Y. Du, J. H. Rasmussen, and M. F. Rasmussen, "SARUS: A synthetic aperture real-time ultrasound system," IEEE Trans. Ultrason., Ferroelec., Freq. Contr., vol. 60, no. 9, pp. 1838-1852, 2013.

[12] A. P. G. Hoeks, M. Hennerici, and R. S. Reneman, "Spectral composition of Doppler signals," Ultrasound Med. Biol., vol. 17, pp. 751-760, 1991.

[13] C. A. Villagomez-Hoyos, "Synthetic aperture vector flow imaging," Ph.D. dissertation, Technical University of Denmark, 2016. 